\title{
Efficacy of gefitinib or erlotinib in patients with squamous cell lung cancer
}

\author{
Zhengbo Song ${ }^{1,2}$, Yiping Zhang ${ }^{1,2}$
}

\begin{abstract}
${ }^{1}$ Department of Chemotherapy, Zhejiang Cancer Hospital, Hangzhou, China
2Key Laboratory Diagnosis and Treatment Technology on Thoracic Oncology, Zhejiang Province, Hangzhou, China
\end{abstract}

Submitted: 3 September 2012

Accepted: 27 December 2012

Arch Med Sci 2015; 11, 1: 164-168

DOI: 10.5114/aoms.2013.39234

Copyright @ 2015 Termedia \& Banach

\author{
Corresponding author: \\ Yiping Zhang MD \\ Department of Chemotherapy \\ Zhejiang Cancer Hospital \\ 38 Guangji Road 310022 \\ Hangzhou, P.R. of China \\ Phone: +8657188122182 \\ Fax: +8657188122188 \\ E-mail: \\ yipingzhang@yahoo.cn
}

\begin{abstract}
Introduction: The aim of this study was to evaluate the feasibility of epidermal growth factor receptor-tyrosine kinase inhibitor (EGFR-TKI) and EGFR mutation frequency in advanced squamous cell lung cancer based on the data from our hospital.

Material and methods: The clinical data of 102 patients with advanced squamous cell lung cancer who were admitted to Zhejiang Cancer Hospital from January 2007 to December 2011 were retrospectively analyzed. Survival analysis was evaluated by the Kaplan-Meier method. The EGFR mutations were assessed in some of the patients using the pyrosequencing assay.

Results: Nine patients (8.8\%) showed a partial response (PR), 28 (27.5\%) achieved stable disease (SD), and 65 (63.7\%) had progressive disease (PD). The disease control rate was $36.3 \%$ and the median progression-free survival (PFS) was 1.93 months (95\% Cl: 1.57-2.29). The PFS in patients who obtained disease control in the prior TKI was 8.63 months and 1.37 months in the disease progression cases $(p<0.001)$. No statistical differences in PFS were observed between gefitinib and erlotinib (2.0 months vs. 1.87 months, $p=0.76)$. The toxicities associated with EGFR-TKI were generally acceptable. In 74 patients with adequate specimens for molecular analysis, 4 (5.4\%) had EGFR mutations (2 with deletions within exon 19 and 2 with L858R mutation in exon 21).

Conclusions: The EGFR-tyrosine kinase inhibitor seems to be a potential therapeutic option for treatment of advanced squamous cell lung cancer patients. Erlotinib and gefitinib had a similar efficacy in advanced squamous cell lung cancer. The frequency of EGFR mutation was about $5.4 \%$ in our single hospital data.
\end{abstract}

Key words: squamous cell lung cancer, erlotinib, gefitinib, efficacy.

\section{Introduction}

Lung cancer is the leading cause of cancer-related death world-wide and squamous cell lung cancer (SCC) accounts for about $30 \%$ of all the cases [1]. There are only a few treatment options for patients with lung SCC beyond standard platinum doublet chemotherapy. Gefitinib, an oral small molecule agent that inhibits epidermal growth factor receptor (EGFR) tyrosine phosphorylation, is the first targeted agent to be approved for the treatment of patients with advanced non-small-cell lung carcinoma (NSCLC), which has demonstrated clinical efficacy in NSCLC [2-4]. Erlotinib, another EGFR-tyrosine kinase inhibitor (TKI), also has shown a survival benefit in second-line or third-line treatment for advanced $\operatorname{NSCLC}[5,6]$. 
Due to the low frequency of EGFR mutation of SCC, a low percentage of patients enrolled in the clinical trials had SCC; thus the efficacy of EGFR-TKI for these patients is not well known. The disease control rate ranged from $27.2 \%$ to $40.6 \%$ according to the previous studies and the frequency of EGFR mutation was between $0 \%$ and $15 \%$ [7-12].

Therefore, we decided to investigate the feasibility and safety of EGFR-TKI in advanced SCC and detect the frequency of EGFR mutation in part of our patient group.

\section{Material and methods}

\section{Patient eligibility}

One hundred and two consecutive, unselected advanced squamous cell lung cancer patients, who were admitted to Zhejiang Cancer Hospital from January 2007 to December 2011, were included in our study. Squamous cell lung cancer staging was performed for all the patients according to the $7^{\text {th }}$ TNM classification. Inclusion criteria were as follows: (1) pathologically proven primary stage IIIB or IV squamous cell lung cancer; (2) the disease recurrence was confirmed using chest computed tomography $(\mathrm{CT})$, brain magnetic resonance imaging (MRI) and bone scan as well as ultrasound examination and/or CT of the abdomen; (3) without any local treatment such as radiotherapy or interventional therapy during the period of gefitinib or erlotinib therapy; (4) at least one measurable lesion and an Eastern Cooperative Oncology Group performance status of 0 to 3.

\section{Response evaluation}

All patients were followed up every $8 \pm 1$ weeks with imaging examination (chest X-ray or computed tomography - CT) during treatment with EGFRTKIs or were evaluated early when significant tumor progression appeared. Objective tumor responses were evaluated according to the Response Evaluation Criteria in Solid Tumors (RECIST 1.1). Objective tumor responses included complete response (CR), partial response (PR), stable disease (SD) and progressive disease (PD). Disease control rate (DCR) was defined as the addition of objective response and stabilization.

\section{Epidermal growth factor receptor mutation examination method}

Epidermal growth factor receptor mutation was performed using pyrosequencing assay methods with formalin-fixed paraffin embedded archival tissue blocks. Epidermal growth factor receptor mutation analysis was performed using sequencing as previously described [13]. Exons 18-21 of EGFR were examined following the standard protocol.

\section{Toxicity evaluation}

The toxicity profile of EGFR-TKI was assessed by reviewing medical records including skin rash, diarrhea, liver toxicity, and radiological evidence of interstitial pneumonitis. Severity of adverse reactions was determined based on the requirements of dosage reduction or discontinuation of EGFR-TKI. All such toxicities were evaluated according to the National Cancer Institute Common Toxicity Criteria version 3.0 (CTC3.0).

\section{Follow-up}

All the patients were to be evaluated for tumor response and PFS. Follow-up rate was $100 \%$. The last follow-up date was June 1, 2012.

\section{Statistical analysis}

The $\chi^{2}$ test was applied to elucidate the differences between different treatment arms. Progression-free survival (PFS) encompassed the time from the first day of TKI treatment to documented progression or death from any cause, or until the date of the last follow-up visit for patients who were still alive and who had not progressed. Survival analysis was conducted with a Kaplan-Meier analysis and log-rank test. A value of $p$ of less than 0.05 was regarded as statistically significant. All statistical tests were analyzed using the computer software SPSS version 16.0 (SPSS Inc, Chicago, IL, USA).

\section{Results}

\section{Patient characteristics}

A total of 769 patients with NSCLC were treated with either gefitinib or erlotinib between January 2007 to December 2011. Of these, there were 102 patients $(13.3 \%)$ with SCC. There were 37 patients (36.3\%) in the gefitinib treatment group and 65 patients (63.7\%) in the erlotinib treatment group (Table I). The median age was 58.0 years (range $35-76$ ) and there were 74 male patients (72.5\%). The characteristics of all the patients and comparison between the DCR and PD patients are summarized in Table I.

\section{Epidermal growth factor receptor mutation analysis}

Seventy-four patients provided tumor samples for EGFR mutation analysis (19 female and 55 male). The EGFR mutations were identified in 4 (5.4\%) patients ( 2 with deletion in exon 19 and 2 with L858R in exon 21). There was no association between gender or smoking and EGFR mutation (1/19 in female and 3/55 in male; $1 / 13$ in non-smoker and 3/61 in smoker).

\section{Efficacy}

Nine patients achieved PR and 28 had SD. No patients achieved CR. The ORR and DCR were $8.8 \%$ and 
Table I. Characteristics of 102 SCC patients

\begin{tabular}{|lcccc|}
\hline Parameter & $N$ & $\begin{array}{c}\text { DCR } \\
\text { group }\end{array}$ & $\begin{array}{c}\text { PD } \\
\text { group }\end{array}$ & Value of $p$ \\
\hline \begin{tabular}{l} 
Gender: \\
\hline Male
\end{tabular} & 74 & 26 & 48 & \\
\hline Female & 28 & 11 & 17 & \\
\hline PS: & & & & 0.69 \\
\hline O-1 & 83 & 33 & 50 & \\
\hline 2-3 & 19 & 4 & 15 & \\
\hline Median age & 58 & 59 & 58 & \\
\hline Age at diagnosis: & & & & 0.14 \\
\hline <65 years & 80 & 32 & 48 & \\
\hline$\geq 65$ years & 22 & 5 & 17 & \\
\hline Smoking: & & & & 0.32 \\
\hline Yes & 83 & 32 & 51 & \\
\hline No & 19 & 5 & 14 & \\
\hline Regimen: & & & & 0.13 \\
\hline Erlotinib & 65 & 20 & 45 & \\
\hline Gefitinib & 37 & 17 & 20 & \\
\hline Stage: & & & & 0.94 \\
\hline IIIB & 84 & 34 & 60 & \\
\hline IV & 61 & 23 & 38 & \\
\hline Prior chemotherapy: & & & 0.29 \\
\hline 1 & & & & \\
\hline$\geq 2$ & & & & \\
\hline
\end{tabular}

$36.3 \%$, respectively. No factors correlated significantly with DCR (Table I). No statistical differences in PFS were observed between gefitinib and erlotinib (2.0 months vs. 1.87 months, $p=0.76$ ) (Figure 1).

There were 4 patients with EGFR mutation and 70 with wild-type. The PFS was 8.0 months in the EGFR mutation patients, but only 1.53 months in the wild-type patients ( $p=0.235$ ) (Figure 2$)$. The PFS was 1.37 months and 8.67 months in PD patients and DCR patients, respectively $(p<0.001)$. The median overall survival of all the patients was 12.2 months. There was a significant difference between the DCR group and PD group in overall survival time (15.2 months vs. 6.4 months, $p<0.001)$.

\section{Factors affecting PFS in univariate and multivariate analysis}

Results of univariate analysis for PFS of SCC are shown in Table II. There were no factors influencing the PFS, but we observed the prolonging trends in younger than older patients $(p=0.073)$.

A multivariate Cox regression model was constructed with the incorporation of age, sex, per-

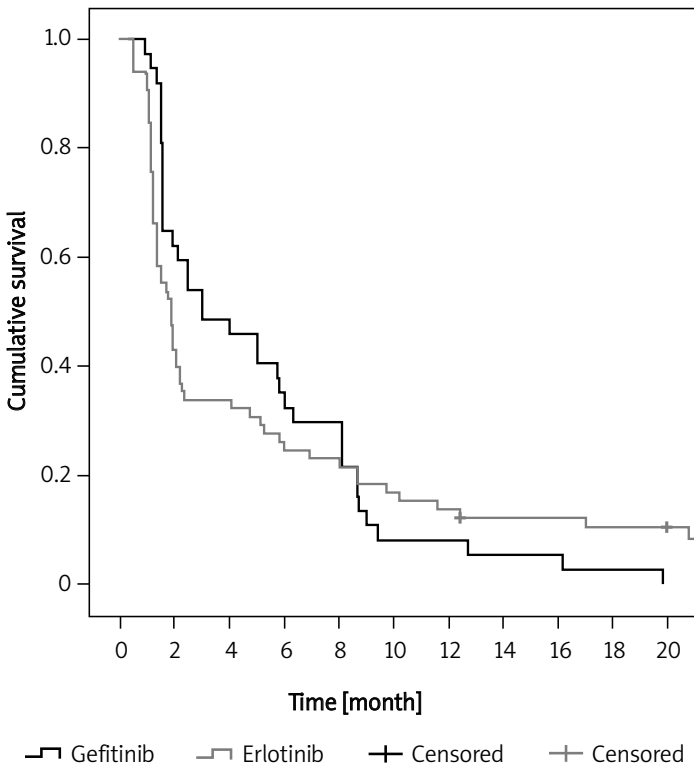

Figure 1. PFS of SCC in gefitinib and erlotinib treatment patients $(p=0.76)$

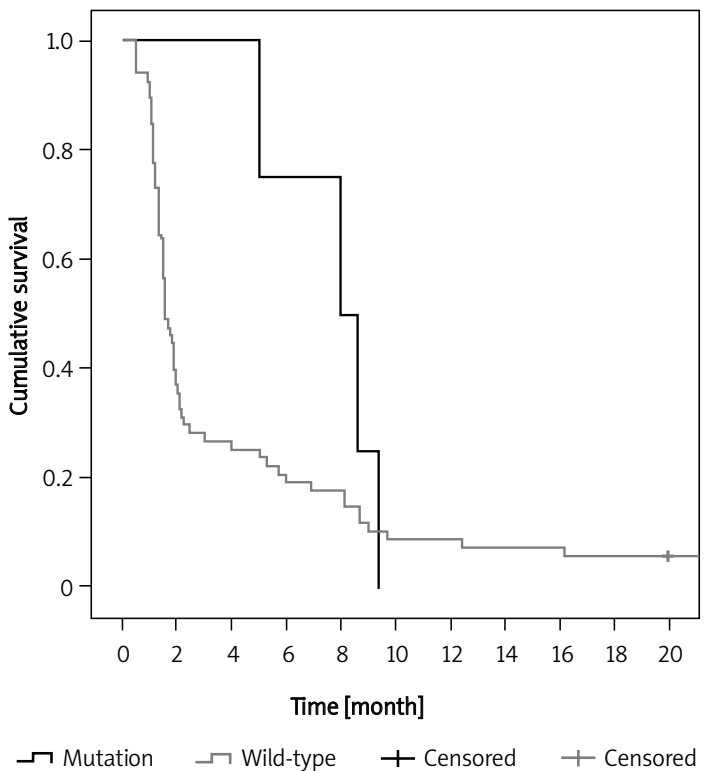

Figure 2. PFS of SCC in EGFR mutation vs. wild-type patients $(p=0.235)$

formance status score (PS), smoking history, TKI type (erlotinib or gefitinib) and prior chemotherapy. No independent prognostic factor was found to influence the PFS (Table III).

\section{Toxicities of tyrosine kinase inhibitor treatment}

Toxicity was evaluated in all patients. The most common adverse event was skin toxicity in 61 patients (59.8\%), including 10 patients with grade 3 . The frequency of rash was more common in erlotinib than gefitinib patients $(66.2 \%$ vs. $48.6 \%$, $p=0.02$ ). Other common toxicities included diarrhea (19 cases), and fatigue (16 cases). Two patients 
demonstrated hepatic function injuries with erlotinib therapy. One dosage reduction occurred.

\section{Discussion}

In this series of patients with SCC who received gefitinib or erlotinib, the objective response and disease control rates were $8.8 \%$ and $36.3 \%$, respectively. The PFS with EGFR mutation and wild-type patients was 8.0 and 1.53 months, respectively. These results support the use of gefitinib or erlotinib as salvage treatment and examination of EGFR mutation in the Asian advanced SCC population. To our knowledge, our study is the largest report that has focused on the efficacy of EGFR-TKIs in patients with EGFR status.

According to the guidelines of the National Comprehensive Cancer Network (NCCN) [14], the EGFRTKIs are recommended as the second-line or thirdline treatment regimen for patients with NSCLC whose ECOG performance status was 0-3 regardless of the histology, which was based on several phase 3 clinical trials such as BR.21 and INTEREST $[15,16]$. However, there is a lack of trials focusing on SCC currently. The ORR of EGFR-TKI treatment was $4.9 \%$ and the DCR was $40.6 \%$ in patients with SCC in the study by Shin et al. [8]. However, the ORR was $17.4 \%$ and DCR was $27.2 \%$ in the Tseng et al. study [7]. Female sex and non-smoking were considered as good factors for the response to EGFR-TKI treatment in adenocarcinoma [17]. Tseng et al. found that there were trends of a higher response rate in females and nonsmokers with erlotinib treatment in SCC patients. In contrast, our study showed that there were no clinical factors associated with response to TKI. The mechanism needs to be investigated in future work.

Epidermal growth factor receptor mutations can be detected in $30-40 \%$ of Asian adenocarcinoma patients; however, mutations can be detected in 5$15 \%$ in the Asian population with SCC $[7-12,18]$. Miyamae et al. found 5 mutation among 89 SCC samples using the PNA-enriched sequencing method; however, only three of these five samples were detected by direct sequencing [12]. There were 4 patients with EGFR mutation in our 74 samples using sequencing assay. The mutation frequency is a little lower than previous reports. The ORR in our data was $8.8 \%$, which is higher than the mutation frequency; some false-negatives with the sequencing may explain the outcome.

A difference in efficacy between the EGFR mutation and wild-type patients was rarely reported in SCC patients. In a pooled analysis by Shukuya et al. including 27 SCC cases with EGFR mutation [19], the PFS ranged from 0.5 month to 11 months (14 patients reported the PFS data and only 2 patients had PFS more than 8 months). The median PFS was 8.0 months in our EGFR mutation patients, which is longer than most of the EGFR mutation patients in
Table II. Univariate analysis of PFS in 102 patients

\begin{tabular}{|c|c|c|c|}
\hline Parameter & PFS & $95 \% \mathrm{Cl}$ & Value of $p$ \\
\hline \multicolumn{3}{|l|}{ Gender: } & 0.82 \\
\hline Male & 1.93 & $1.57-2.29$ & \\
\hline Female & 1.93 & $0.89-2.97$ & \\
\hline \multicolumn{3}{|l|}{ PS: } & 0.79 \\
\hline $0-1$ & 2.17 & $1.61-2.74$ & \\
\hline $2-3$ & 1.67 & $1.28-2.07$ & \\
\hline \multicolumn{3}{|l|}{ Age: } & 0.073 \\
\hline$<65$ years & 2.1 & $1.52-2.68$ & \\
\hline$\geq 65$ years & 1.5 & $0.86-2.14$ & \\
\hline \multicolumn{3}{|l|}{ Smoking: } & 0.32 \\
\hline Yes & 1.53 & $0.17-2.90$ & \\
\hline No & 2.03 & $1.73-2.33$ & \\
\hline \multicolumn{3}{|l|}{ Regimen: } & 0.76 \\
\hline Erlotinib & 1.87 & $0.49-5.5$ & \\
\hline Gefitinib & 2.00 & $1.45-2.29$ & \\
\hline \multicolumn{3}{|l|}{ Stage: } & 0.96 \\
\hline III B & 1.99 & $1.25-2.32$ & \\
\hline IV & 1.93 & $1.12-2.55$ & \\
\hline \multicolumn{3}{|c|}{ Prior chemotherapy: } & 0.65 \\
\hline 1 & 2.10 & $1.19-2.43$ & \\
\hline$\geq 2$ & 1.87 & $1.57-2.29$ & \\
\hline \multicolumn{3}{|l|}{ Mutation: } & 0.235 \\
\hline Yes & 8.0 & $4.44-11.56$ & \\
\hline No & 1.53 & $1.20-1.86$ & \\
\hline
\end{tabular}

Table III. Multivariate analysis of PFS in patients with SCC

\begin{tabular}{|lccc|}
\hline Variables & HR & $\begin{array}{c}95 \% \text { Confidence } \\
\text { interval }\end{array}$ & Value of $p$ \\
\hline Sex & 1.220 & $0.704-2.113$ & 0.478 \\
\hline Age & 1.640 & $0.983-2.736$ & 0.846 \\
\hline PS & 1.173 & $0.693-1.987$ & 0.553 \\
\hline TKI type & 1.145 & $0.746-1.756$ & 0.536 \\
\hline $\begin{array}{l}\text { Prior } \\
\text { chemotherapy }\end{array}$ & 0.817 & $0.512-1.978$ & 0.817 \\
\hline Smoking history & 1.178 & $0.693-1.987$ & 0.272 \\
\hline
\end{tabular}

the Shukuya et al. pooled analysis. Rare mutations (such as exon 20 A763V, exon 21 N826S) were prevalent in the Shukuya et al. study; however, all of our four patients had common mutations ( 2 with exon 19 deletion and 2 with exon 21 L858R), which may partly explain the efficacy difference in our study.

There are somewhat different pharmacological properties between gefitinib and erlotinib in experiments and the clinic [20]. The maximal tolerated dose (MTD) of gefitinib is lower than that of erlotinib. 
Erlotinib has a much better profile of DCR, PFS and OS than gefitinib in the Fan et al. study [21]. However, the efficacy difference was not clear in SCC. The efficacy was similar between erlotinib and gefitinib regardless of the PFS and OS in the current study. Further studies are warranted to examine the difference in the treatment effect between the two drugs.

Advances in personalized treatments with targeted biologic agents, including agents that target mutant kinases such as EGFR and ALK, and the multi-targeting antifolate pemetrexed, are not applicable to SCC. Fortunately, many driving genetics have been presented in SCC recently, and there is growing evidence of their biologic significance. Thus, in the near future, the molecular characterization of patients with SCC will probably be as important as deciphering the molecular genetics of adenocarcinoma. Patients with SCC of the lung harboring specific molecular defects such as DDR2, FDFR1 and PI3K are undergoing clinical trials targeting such molecular defects [22].

The major limitation of the present study is its retrospective nature. In addition, sequencing was used to analyze the EGFR mutations in our patients. It has a lower sensitivity than other methods, and may increase the false-negative rate in the EGFR mutation result. However, with few cases even in limited clinical trials, our retrospective study can also be considered to be meaningful.

In conclusion, a significant proportion of SCC patients would derive a clinical benefit from TKI treatment. The mutation frequency was approximately $5.4 \%$ in our study. Prospective studies with larger cohorts should be conducted to verify the efficacy of TKI in SCC patients and mutation frequency.

\section{Conflict of interest}

The authors declare no conflict of interest.

\section{References}

1. Wahbah M, Boroumand N, Castro C, et al. Changing trends in the distribution of the histologic types of lung cancer: a review of 4439 cases. Ann Diagn Pathol 2007; 11: 89-96.

2. Mok TS, Wu YL, Thongprasert S, et al. Gefitinib or carboplatin-paclitaxel in pulmonary adenocarcinoma. N Engl J Med 2009; 361: 947-57.

3. Maemondo M, Inoue A, Kobayashi K, et al. Gefitinib or chemotherapy for non-small-cell lung cancer with mutated EGFR. N Engl J Med 2010; 362: 2380-8.

4. Mitsudomi T, Morita S, Yatabe Y, et al. Gefitinib versus cisplatin plus docetaxel in patients with non-small-cell lung cancer harbouring mutations of the epidermal growth factor receptor (WJTOG3405): an open label, randomised phase 3 trial. Lancet Oncol 2010; 11: 121-8.

5. Gatzemeier U, Pluzanska A, Szczesna A, et al. Phase II study of erlotinib in combination with cisplatin and gemcitabine in advanced non-small-cell lung cancer: the Tarceva Lung Cancer Investigation Trial. J Clin Oncol 2007; 25: 1545-52.
6. Ciuleanu T, Stelmakh L, Cicenas S, et al. Efficacy and safety of erlotinib versus chemotherapy in second-line treatment of patients with advanced, non-small-cell lung cancer with poor prognosis (TITAN): a randomised multicentre, open-label, phase 3 study. Lancet Oncol 2012; 13: 300-8.

7. Tseng JS, Yang TY, Chen KC, et al. Retrospective study of erlotinib in patients with advanced squamous lung cancer. Lung Cancer 2012; 77: 128-33.

8. Shin HJ, Kim TO, Kang HW, et al. Comparison of therapeutic efficacy of gefitinib and erlotinib in patients with squamous cell lung cancer. Tuberc Respir Dis 2011; 71: 15-23.

9. Kowalski DM, Krzakowski M, Ramlau R, et al. Erlotinib in salvage treatment of patients with advanced non-small cell lung cancer: results of an expanded access programme in Poland. Contemp Onkol (Pozn) 2012; 16: 170-5.

10. Huang SF, Liu HP, Li LH, et al. High frequency of epidermal growth factor receptor mutations with complex patterns in non-small cell lung cancers related to gefitinib responsiveness in Taiwan. Clin Cancer Res 2004; 10: 8195-203.

11. Tomizawa $\mathrm{Y}$, lijima $\mathrm{H}$, Sunaga $\mathrm{N}$, et al. Clinicopathologic significance of the mutations of the epidermal growth factor receptor gene in patients with non-small cell lung cancer. Clin Cancer Res 2005; 11: 6816-22.

12. Miyamae $Y$, Shimizu K, Hirato J, et al. Significance of epidermal growth factor receptor gene mutations in squamous cell lung carcinoma. Oncol Rep 2011; 25: 921-8.

13. Chou TY, Chiu CH, Li LH, et al. Mutation in the tyrosine kinase domain of epidermal growth factor receptor is a predictive and prognostic factor for gefitinib treatment in patients with non-small cell lung cancer. Clin Cancer Res 2005; 11: 3750-7.

14. National Comprehensive Cancer Network (NCCN). National Comprehensive Cancer Network: your best resource in the fight against cancer [Homepage]. Fort Washington, PA: NCCN; c2011 [cited 2011 July 20]. Available from: http://www.nccn.org/index.asp.

15. Shepherd FA, Pereira JR, Ciuleanu T, et al. Erlotinib in previously treated non-small-cell lung cancer. N Engl J Med 2005; 353: 123-32.

16. Thatcher N, Chang A, Parikh P, et al. Gefitinib plus supportive care in previously treated patients with refractory advanced non-small-cell lung cancer: results from a randomized, placebo-controlled, multicenter study (Iressa Survival Evaluation in Lung Cancer). Lancet 2005; 366: 1527-37.

17. Park K, Goto K. A review of the benefit-risk profile of gefitinib in Asian patients with advanced non-small-cell lung cancer. Curr Med Res Opin 2006; 22: 561-73.

18. Park SH, Ha SY, Lee J, et al. Epidermal growth factor receptor mutations and the clinical outcome in male smokers with squamous cell carcinoma of lung. J Korean Med Sci 2009; 24: 448-52.

19. Shukuya T, Takahashi T, Kaira R, et al. Efficacy of gefitinib for non-adenocarcinoma non-small-cell lung cancer patients harboring epidermal growth factor receptor mutations: a pooled analysis of published reports. Cancer Sci 2011; 102: 1032-7.

20. Comis RL. The current situation: erlotinib (Tarceva) and gefitinib (Iressa) in non-small cell lung cancer. Oncologist 2005; 10: 467-70.

21. Fan WC, Yu CJ, Tsai CM, et al. Different efficacies of erlotinib and gefitinib in taiwanese patients with advanced non-small cell lung cancer: a retrospective multicenter study. J Thorac Oncol 2011; 6: 148-55.

22. Perez-Moreno P, Brambilla E, Thomas R, et al. Squamous cell carcinoma of the lung: molecular subtypes and therapeutic opportunities. Clin Cancer Res 2012; 18: 2443-51. 